\title{
Electrical impedance tomography based image reconstruction and feto-maternal monitoring in pregnancy
}

\author{
Sharvan Kumar Pahuja ${ }^{1 *}$, Sneh Anand ${ }^{1}$, Amit Sengupta ${ }^{1,2}$ \\ ${ }^{1}$ Centre for Biomedical Engineering, Indian Institute of Technology Delhi; Hauz Khas, New Delhi, India; ${ }^{*}$ pahujas@gmail.com; \\ snehanand@rediffmail.com; \\ ${ }^{2}$ OBGYN, Mumbai, India; senguptaamit@hotmail.com
}

Received 20 January 2011; revised 5 April 2011; accepted 15 June 2011.

\begin{abstract}
Standard methods of monitoring the fetus and maternal health during labor are cardiotocogram, tocography, ultrasound and magnetocardiograpghy. These methods have some limitations in real time continuous monitoring and cause some degree of inconvenience to the patient and demand special attendance of the obstetrician also these methods cannot be used for continuous monitoring of the fetal well being. To overcome the limitations of above techniques, a non-invasive bioimpedance measuring method is proposed. This technique helps in monitoring and recording of the electrical field distribution of a closed object. The output variation on the outer surface is likely to provide information because of fetal movements and related physiological parameters. It will also help in the development of Electrical Impedance Tomography based imaging technique for a closed body system with special reference to fetal monitoring in-utero during pregnancy and labor. Also we have developed the data acquisition system of 16 electrodes with software for image reconstruction.
\end{abstract}

Keywords: Impedance Plethysmography; Electrical Impedance Tomography; Phantom

\section{INTRODUCTION}

Feto-maternal monitoring has become a very important tool to control a fetal health throughout the pregnancy and during labor. The vital parameters like fetal ECG, uterine contraction, fetal movements, and growth of the fetus, amniotic fluid volume and position of placenta have to be in good condition for the fetus health. Fetus in situ initi- ates compensatory protective mechanism which enables it to survive from any altered state/ abnormalities (such as cerebral palsy, placenta previa, asphyxia, respiratory and the growth) $[12,13]$ that may occur both during normal as well as abnormal pregnancy. These abnormalities could be avoided by the timely diagnosis by continuously monitoring the fetus and mother by the use of the instruments. It is imperative to monitor vital parameters at a regular interval throughout pregnancy and during labor for feto-maternal well being and for better outcome [14]. Techniques and Instrumentations have been developed to monitor various parameters and for feto-maternal wellbeing.

Cardiotocogram (CTG) is a standard method for monitoring of fetal heart rate (FHR) and the uterine contractions (UC) in the later stage of the pregnancy. By analysis and appropriate interpretation of changes in the CTG obstetrician are able to prevent still birth or asphyxia. Other available techniques such as external and internal tocography (TOCO) [17], Ultrasound [16] and Magnetocardiograpghy [17] have also been in used for monitoring of vital parameters of the fetus and mother [18]. The growth of the fetus and in understanding of the anatomy and functions of an organ or a physiological process, various invasive and non-invasive imagining techniques are available. All medical modalities rely on ionizing radiation to produce images of structure and function through a variety of mechanism but the main biological risk is cancer when imaging in utero. Ultrasound is widely used for imaging of the fetus which has some limitations like long term monitoring is not possible and demands well trained experience ultrasonographer for data acquisition.

Despite their scientific perfection all these methods cause some degree of inconvenience to the patient and demand special attendance of the obstetrician. Also these methods cannot be used for continuously monitoring of 
the feto-maternal parameters because they are bulky, expensive, time consuming and need skilled personnel.

To overcome the limitations, here electrical impedance monitoring based instrument is proposed. This technique helps in continuously recording and monitoring noninvasively of inside electrical field distribution in term of voltage for a given current. The output voltage on the abdomen surface of a pregnant lady is likely to provide information regarding fetal movement and its growth. The method has already been used in measurement of bioimpedance. An electrical impedance plethysmograph based instruments have been using in monitoring of blood flow in the heart [Nyboer]. Nicander et al. and Skourou et al. have shown that a tissue's impedance signature may be more sensitive to presence of tumor tissues than conventional imaging technique of computed tomography and ultrasound. Also the technique has already been used for monitoring respiration [Bake], measurement of cardiac output [Kubicek]. Further the concept of Electrical Resistive Tomography was proposed independently by Henderson and Webster for medical imaging. Kim has applied impedance technique for measurement of various physiological events. Each organ is an aggregate of many different cells held together by intracellular supporting structures. The conductivity of human tissues varies from cerebrospinal fluid to bone in very large difference. The range is $15 \mathrm{mS} / \mathrm{cm}$ to $0.067 \mathrm{mS} / \mathrm{cm}$ [4]. The Table 1 shows the conductivities of various tissues assumed for $50 \mathrm{~Hz}$ [Cech]. As shown in table the distribution of conductivity inside the body shows good contrast hence good electrical conductivity images can be produced of these distributions.

\section{THEORETICAL MODEL AND MEASURING METHODOLOGY}

Figure 1 shows pictorial representation of current distribution and equi-potential lines on the surface of the

Table 1. shows the conductivities of various tissues assumed for $50 \mathrm{~Hz}$.

\begin{tabular}{cc}
\hline Tissue & Conductivity $\left(\mathbf{S ~ m}^{\mathbf{- 1}}\right)$ \\
\hline Bone & 0.0201 \\
Fat & 0.0196 \\
Meart & 0.0827 \\
Suscle & 0.233 \\
Soft tissue of fetus & 0.0201 \\
Spinal cord & 0.216 \\
Uterus & 0.0227 \\
\hline
\end{tabular}

cell contains homogenous electrolyte solution. The high frequency low AC current is applied between electrodes $\mathrm{I}_{1}, \mathrm{I}_{2}$ which gets uniformly distributed and the resulting voltage is measured between electrodes $V_{1}$ and $V_{2}$. Figure 2 shows the complete experimental setup. The excitation current is applied into the subjects between Q-S electrodes in the range of $(0.8 \mathrm{~mA}-1 \mathrm{~mA} \mathrm{AC})$ at 20 $100 \mathrm{KHz}$. The voltage produced between P-R electrodes is amplified and fed to PC using data acquisition system. This set up is act as a bridge configuration as shown Figure 3. Any variation inside (because of fetal movement) will affect the abdomen surface of the pregnant woman. This can be measured non-invasively in term voltage. The current and voltage electrodes are switched through different combinations so that the transfer impedance is measured for different position of applied currents.

The same principle is used in Electrical Impedance Tomography (EIT) in which an image of the conductivity distribution within a cross-section of the body is obtained. The technique exploits the electrical characteristics of the tissues by measuring voltages induced on the electrodes attached to the surface when the currents are passed between them. The instrument is makes impedance measurement using 16 electrodes on the surface of the body. A current is passed between pairs of electrodes (QS). The voltage developed between remaining pairs of electrodes is than measured sequentially by an array of instrument amplifiers and fed to data acquisition system for recording and further analysis. The general schematic diagram of the data acquisition is shown in Figure 4.

\section{METHODS AND MATERIAL REQUIRED}

Presently some preliminary work is being carried out

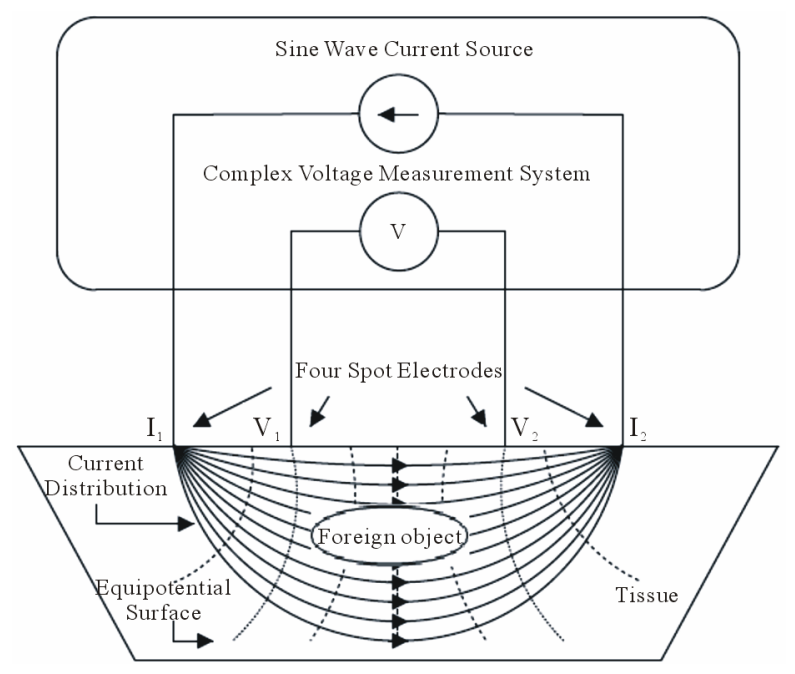

Figure 1. Four-electrode measurement system. 


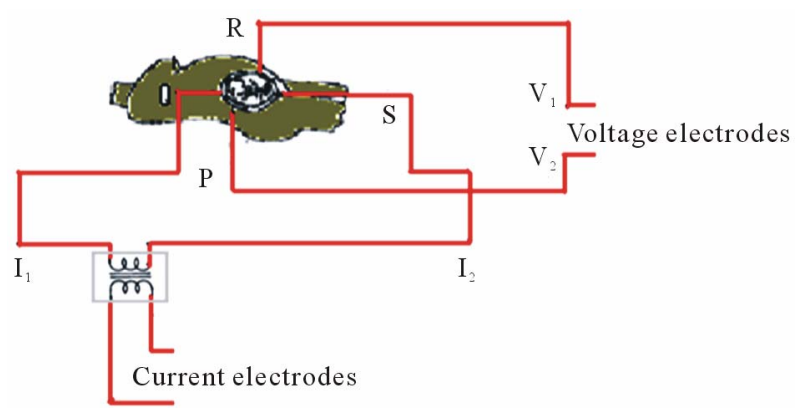

Figure 2. Block Diagram of impedance measuring system.

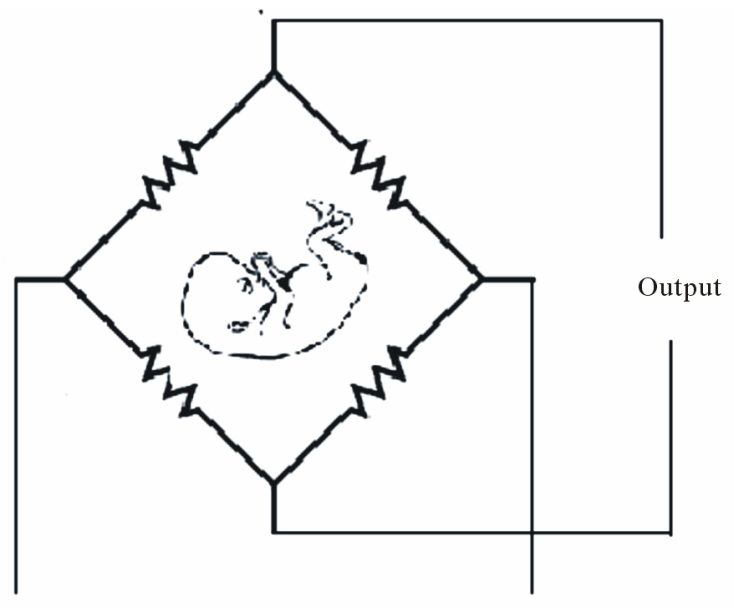

Current in range of $0.8-1 \mathrm{~mA}$ at $200-100 \mathrm{KHz}$

Figure 3. Equivalent bridge diagram in case of fetal movements.

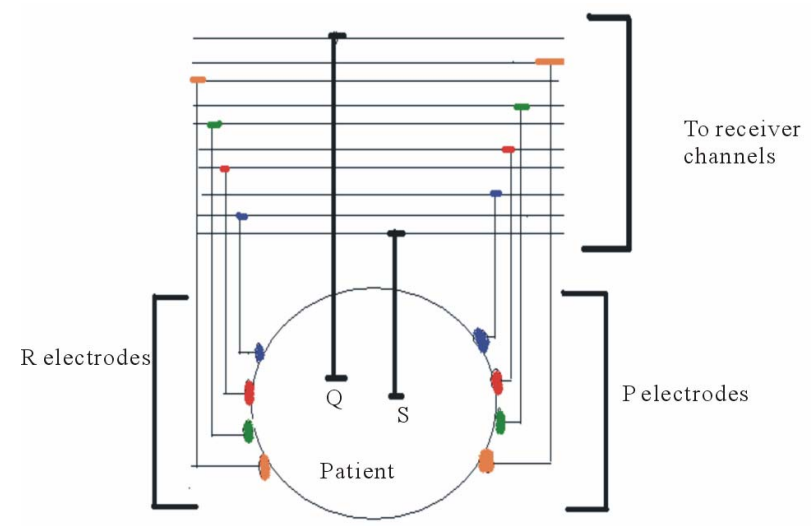

Figure 4. Block diagram of the real-time EIT configuration.

by conducting experiments on a phantom uterus and fetus. Vital parameters have been recorded using external tocography and impedance measurement system. And images have been reconstructed by image reconstruction algorithm.

Advanced experimental studies will be carried out to develop and standardized the instrumentation. The actual clinical applicability shall be checked at later stages during clinical trials (on human and animals). The experiments will be conducted on both pregnant women (with gestation age 25 - 36 weeks) and pregnant animals for the clinical trials to establish the relationship between fetal movement and immature fetal autonomic nervous system from the recorded data.

\section{MODEL BASED EXPERIMENTS}

Presently some preliminary work is being carried out by conducting experiments on a phantom uterus and fetus. The work is validated by conducting some experiments by taking papaya and plastic box as phantom. First experiment is performed on phantom papaya. Papaya is chosen as it imitates the properties of human's uterus. Second experiment is conducted on plastic box.

\subsection{Case Study 1}

Figure 5 shows papaya, watermelon, melon and coconut as phantom where we could observe the variation when the ac current was supplied through two electrodes. The electrode configuration is different in each case.

\subsection{Case Study II}

Second experiment was conducted on plastic container. The set up is as shown in Figure 6. The input here is 4 volts at frequency of $40 \mathrm{KHz}$. The output was measured between other pairs of electrode by opposite method of data acquisition.

\subsection{Case study II}

The electrical conductivity distribution was measured for a closed object using data acquisition methods. Data was collected by applying ac current pattern in the range of $1-5 \mathrm{~mA}$ at a frequency of $40 \mathrm{KHz}$ to the phantom papaya through the two conducting electrodes and then inside conductivity is measured on the papaya surface in term of voltage between remaining pairs of the electrodes using opposite method.

The current-voltage distribution relationship of the applied current and measured voltage is determined by Poisson's or Laplace's equation with given boundary conditions measured at the surface. An image of conductivity distribution was obtained for the closed object using appropriate image reconstruction algorithm. Software is also developed for creating meshes, nodes and for solving the independent equations for finding the pixel intensity of an image using iterative methods. Figure 7 shows the complete experiment set up and the images reproduced by using image reconstruction algorithm. 


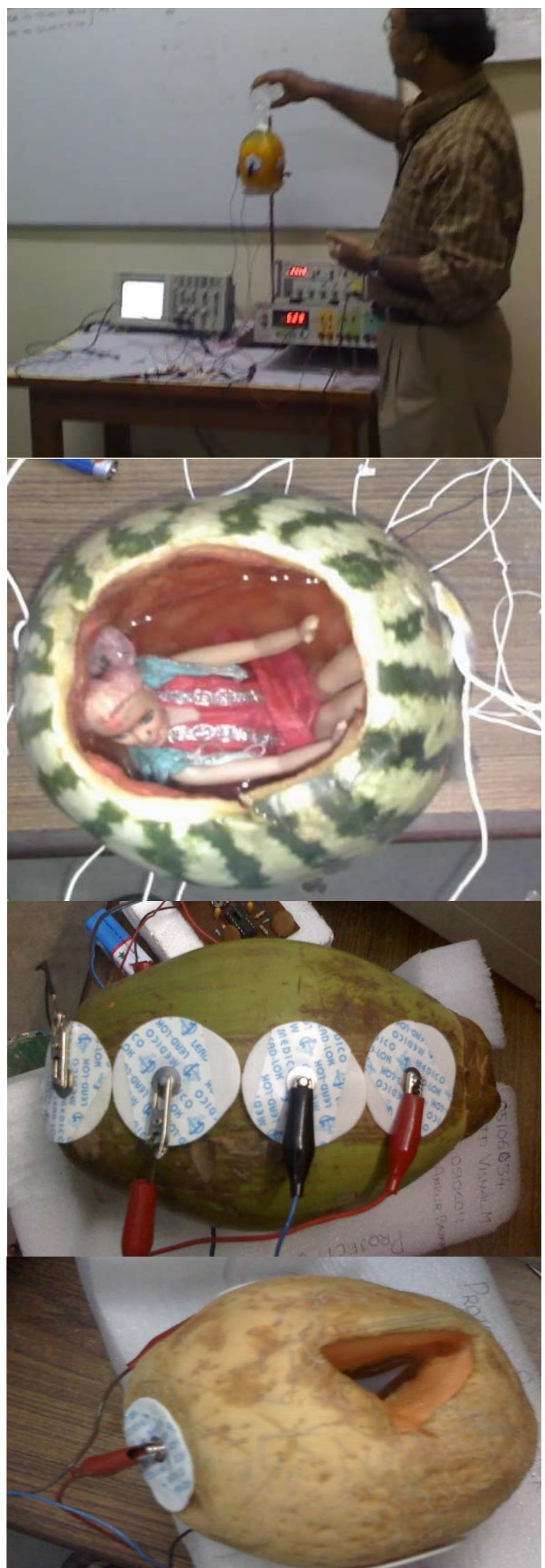

Figure 5. Experimental Setup with different phantom.

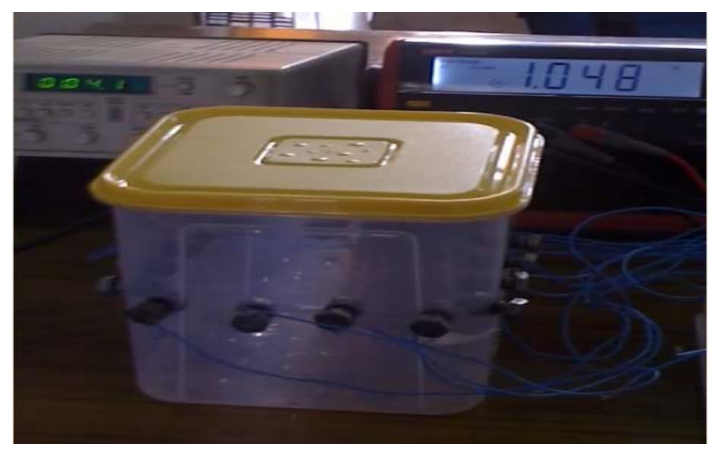

Figure 6. Complete experimental setup with plastic container as model.

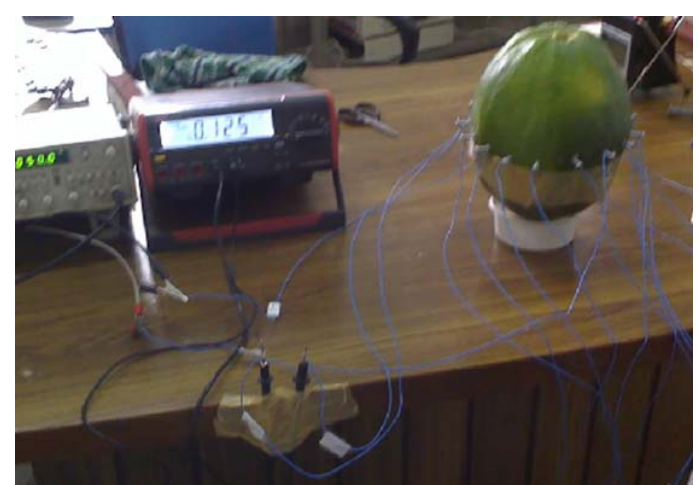

(a)

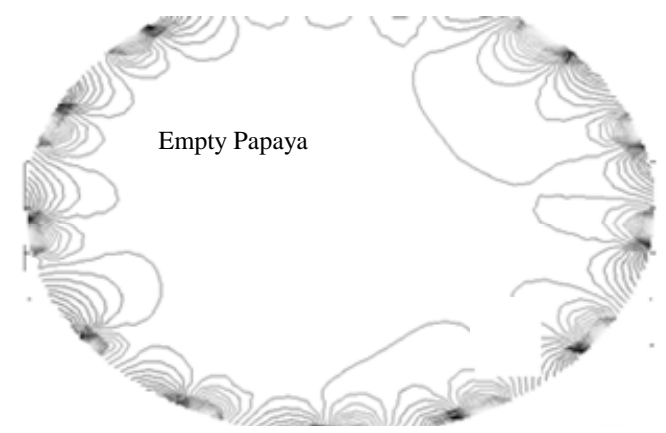

(b)

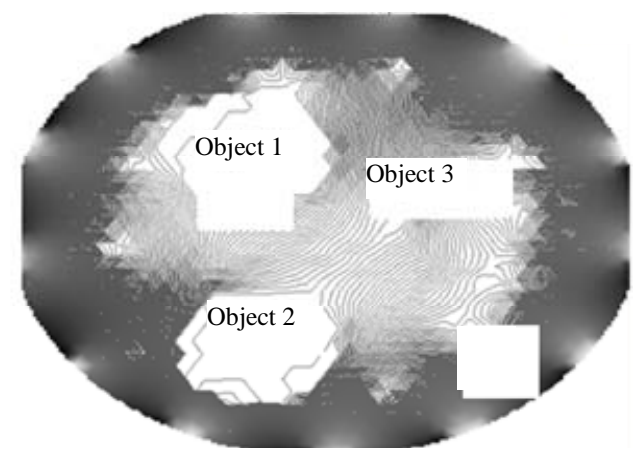

(c)

Figure 7 (a) Experiment setup (b) The 2-D image of an empty papaya (c) The 2-D image of the phantom papaya with objects inside.

Openly accessible at http://www.scirp.org/journal/HEALTH/ 


\section{CONCLUSION}

The new technique being proposed in this work is noninvasive, user friendly, economical and for mass health care which can be used by the poor community and the basic health worker. Also the novel approach will help in defining the morphological and physiological changes occurring inside the body using EIT as a functional non invasive imaging tool.

\section{REFERENCES}

[1] Stuchly, M.A. and Gandhi, O.P. (1994) Induced electric currents in models of man and rodents from $60 \mathrm{~Hz}$ magnetic fields. Engineering, 41, 1088-1093,

[2] Gordon, A.S., Strauss, J. and George, A.M. (1965) Electrical impedance of isolated amnion. Biophysical Journal, 5, 855-865.

[3] Hoe, Y., Gurewitsch, E.D., Shaahinfar, A., Somponnat, E.S., Ruffner, S.M., Kenny, H.S. and Allen, H. (2004) Measuring bioimpedance in the human uterine cervix: Towards early detection of preterm labor. 1-5.

[4] Steer, C.M. and Hertsch, G.J. (1950) Electrical activity of the human uterus in labor: The electrohysterograph. American Journal of Obstetrics \& Gynecology, 59, 2540.

[5] Katz, M., Gill, P.J., and Newman, R.B. (1986) Detection of preterm labor by ambulatory monitoring of uterine activity: A preliminary repot. Obstetrics \& Gynecology, 68, 773-778.

[6] Lucidi, R.S., Blumenfeld, L.A. and Chez, R.A. (2000) Cervimetry: A review of methods for measuring cervical dilation during labor. Obstetrical \& Gynecological Survey, 55, 312-320.

[7] Friedman, E.A. (1956) Cervimetry: An objective method for the study of cervical dilation in labor. American Journal of Obstetrics \& Gynecology, 71, 1189-1193.

[8] Myoung, H.C., Tzu, K., Gary, D.I. and Jonathan, C.N. (2007) A reconstruction algorithm for breast cancer imaging with electrical impedance tomography in mammography geometry. Biomedical Engineering, 54, 700710 .
[9] Smith, R.W., Ian, L. and Brian, H.B. (1995) A real-time electrical impedance tomography system for clinical use-design and preliminary results. Biomedical Engineering, 42, 133-140.

[10] Ritsuko, Y., Kyousuke, T., Toru, F. and Takeshi, M. (2003) Bioelectrical impedance analysis in the clinical management of preeclamtic women with edema. Journal of Perinatal Medicine, 31, 275-280.

[11] Leman, H., Catherine, M. and Jean, G. (1999) Use of the electrohysterogram signal for characterization of contractions during pregnancy. IEEE Transactions on Biomedical Engineering, 46, 672-680.

[12] Gaysen, A., Dua, S.K., Sengupta, A. and Nagchoudhuri, Effect of non-linearity doppler waveforms through novel model. Biomedical Engineering Online, 1-13.

[13] Ritsuko, Y., Kyousuke, T., Toru, F. and Takeshi, M. (2003) Bioelectrical impedance analysis in the clinical management of preeclamtic women with edema. Journal of Perinatal Medicine, 31, 275-280.

[14] Katz, M., Gill, P.J. and Newman, R.B. (1986) Detection of preterm labor by ambulatory monitoring of uterine activity: A preliminary repot. Obstetrics and Gynecology, 68, 775-778.

[15] Goovaerts, H.G., Rompelman, O. and Gejin, H.P. (1989) A transducer for detection of fetal breathing movement. IEEE Transactions on Biomedical Engineering, BME-36, 471-478. doi:10.1109/10.18754

[16] Magann, E., Perry, K.G., Chauhan, S.P., Anfanger, P.J., Neil, R.N., Whitworth, S. and Morrison, J.C. (1998) The accuracy of ultrasound evaluation of amniotic fluid volume in singleton pregnancies: The effect of operator experience and ultrasound interpretative technique. Journal of Clinical Ultrasound, 25, 249-253.

[17] Salustri, C., Barbate, G. and Porcaro, C. (2005) Fetal Magnetocardiographic Signal Extraction by Signal Subspace: Blind Source Separation. IEEE Transactions on Biomedical Engineering, 52, 1140-1142. doi:10.1109/TBME.2005.846708

[18] Steer, C.M. and Hertsch, G.J. (1950) Electrical activity of the human uterus in labor: The electrohysterograph. American Journal of Obstetrics \& Gynecology, 59, 2540. 